Ueber den Einfluss der Temperatur auf die Respiration der Kaltblüter. 73

(Physiologisches Laboratorium in Bonn.)

\title{
Ueber den Einfluss der Temperatur auf die Respiration der Kaltblüter.
}

(Einleitung zu der folgenden Experimentalarbeit von Herrn Hugo Schulz.)

Von

E. Pffüger.

In der gelehrten Welt herrscht eine erfreuliche Uebereinstimmung über die Thatsache, dass die Kaltblüter einen um so energischeren Stoffwechsel haben, je höher die Temperatur ihrer Umgebung, resp. ihres Körpers ist. Als ich zum Behuf der ausführlichen Ausarbeitung meiner Theorie der Lebensprocesse die einzelnen Literaturangaben genauer kritisch untersuchte, gelangte ich zu der Einsicht, dass unsere Auffassungen sich bisher mehr durch eine Art wissenschaftlichen Instinctes, als durch Beweise haben leiten lassen. Denn die Beweise für die Ansicht, dass der Stoffwechsel der Kaltblüter mit der Temperatur wachse, stehen auf sehr schwachen Füssen.

Die beiden wichtigsten Untersuchungen, auf welche jene Ansicht sich gewöhnlich zu stützen pflegt, sind die von Marchand und Moleschott. Beide Forscher weichen in ihren Resultaten sehr wesentlich von einander ab. Denn nach Marchand ${ }^{1}$ ) ist der Stoffwechsel der Amphibien keineswegs der Temperatur proportional, sondern hat ungefähr bei $6-14^{\circ} \mathrm{C}$, ein Maximum; eine Differenz von $6-7^{0}$ macht hier nach ihm wenig Einwirkung; erst wenn die Temperatur bis zu dem Eispunkte sinke und ebenso, wenn sie über 28 bis $30^{\circ}$ C. steige, nehme der Sauerstoffverbrauch und die Kohlensäurebildung ab. Nach Moleschott, der als eigentlicher Gewährsmann gewöhnlich citirt zu werden pflegt, ist aber die Kohlensäurebildung der Temperatur proportional.

1) Marehand, Journ. f. prakt. Chemie. Bd. XXXIII. 152. 
Marchand's Angaben sind falsch, und die Ursache seiner Irrthümer liegt, wie ich finde, zum Theil sicher in chemischen Fehlern, obwohl er ein Chemiker war. Er leitete atmosphärische Luft durch einen abgeschlossenen Raum, in dem sich die Frösche befanden und liess die vorher getrocknete Luft dann durch Kalilauge passiren, deren Gewichtszunahme die Menge der ausgeschiedenen Kohlensäure ergibt. Die den Thieren zugeführte atmosphärische Luft suchte er von Kohlensäure zu befreien, indem er sie über trockne Kalistangen streichen liess, wobei sie bekanntlich, wie jeder mit diesen Verhältnissen Vertraute weiss, ihre Kohlensäure nur ganz unvollständig verliert. Da er nun sehr grosse Mengen atmosphärischer Luft, die aber wenig von ihrer Kohlensäure verloren hatte, den Thieren zuführte, so ist die Kohlensäure, welche angeblich von den Thieren producirt ist, wirklich nur zu einem unbekannten Theil von diesen producirt. Da Frösche überhaupt nicht viel erzeugen, so kommt dieser Fehler in Betracht. Weil er endlich den Sauerstoff indirect berechnet - auf Grund der Kohlensäurewerthe, welche falsch sind, so sind auch seine Sauerstoffwerthe nicht $\mathrm{zu}$ benutzen.

Die Versuche Moleschott's ${ }^{1}$ ) leiden an anderen, aber wesentlichen Uebelständen. Wenn er z. B. den Stoffwechsel bei $30^{\circ} \mathrm{C}$. bestimmen will, so bedeutet diess, dass er einen Frosch, dessen innere Körpertemperatur niemals gemessen wird, in einen Raum setzt, in dem ein aufgehängtes Thermometer $30^{\circ} \mathrm{C}$. zeigt. Der Frosch hat aber vielleicht nur $20^{\circ} \mathrm{C}$. am Ende des Versuches erreicht, der bei Moleschott immer nur eine Stunde dauert. Auch nach Ablauf dieser Stunde, in der der Stoffwechsel bei $" 30^{\circ}$ C. " beobachtet wurde, hat niemals Moleschott gemessen, welche Temperatur der Frosch selbst denn nun eigentlich gehabt habe. Das wird sehr abhängen von der Temperatur, die er am Anfang des Versuches hatte. Es ist übrigens bekannt, dass die Frösche innerhalb gewisser Grenzen durch Verdunstung von Wasser ihre Temperatur in der Hitze niedrig zu halten vermögen. Man weiss also bei Moleschott die Tem. peratur des Froschkörpers nicht.

Ein zweiter wesentlicher Uebelstand ist folgender. Wenn ich einen Frosch aus einem kühlen Behälter in einen sehr warmen Raum

1) Jac. Moleschott, Ueber den Einfluss der Wärme auf die Kohlensä,ureausscheidung der Frösche in den Untersuchungen zur Naturlehre von Jac. Moleschott. 1857. p. 315. Bd. II. 
Ueber den Einfluss der Temperatur auf die Respiration der Kaltblüter. 75

bringe, so steigere ich die Temperatur seines Körpers - gleichgültig, wie viel. Nun ist es aber bekannt, dass der Absorptionscoefficient der Kohlensäure mit wachsender Temperatur ausserordentlich abnimmt. 1 Maass Wasser absorbirt bei $0^{0}$ C. 1,7967 ${ }^{1}$ ) Maass Kohlensäure; bei der Temperatur des Blutes der Warmblüter aber, mit der Mol esc h ot t auch experimentirt hat, absorbirt 1 Maass Wasser nur 0,522) Maass Kohlensäure. Daraus folgt also, dass ein Frosch, der aus dem Kalten ins Warme gebracht wird, sofort mehr Kohlensäure aushauchen muss, wenn er auch nicht die Spur mehr producirt. - Ferner: der bei Weitem grösste Theil der Kohlensäure ist im Körper locker gebunden, also in Dissociation. Mit wachsender Temperatur steigt aber die Spannung der lockeren Gasverbindungen auch; folglich muss auch mehr aus diesen Verbindungen stammende Kohlensäure abgegeben.werden. Wer will aber selbst bei unseren heutigen Kenntnissen die möglichen Massen locker gebundener Kohlensäure des thierischen Körpers schätzen, wenn man zu den Carbonaten die riesigen Massen des Calciumcarbonates der Knochen rechnet, welches eine colossale lockere Bindung von Kohlensäure bedingen könnte. Die Anziehung der Bindesubstanz und die grosse Masse der Kalksalze im Knochen könnte die Auflösung des sonst in Wasser löslichen Bicarbonates des Kalks verhindern. - Wenn also ein in's Warme gebrachter Frosch nun mehr Kohlensäure ausgiebt, so beweist diess Nichts für eine Mehrbildung.

Die umgekehrten Betrachtungen sind zu machen, wenn ein Frosch, in's Kalte gebracht, weniger Kohlensäure ausscheidet; er muss es auch dann eine Zeit lang thun, wenn er genau soviel als vorher im Warmen producirt.

Wenn man den Versuch also damit anfängt, dass man ein Thier plötzlich erwärmt oder abkühlt, so wird der Fehler um so bedeutender, je kürzere Zeit der Versuch dauert. Denn es addirt sich algebraisch zu der wirklich producirten Menge noch diejenige, Menge der Kohlensäure, welche durch die Veränderung der Temperatur des Froschkörpers entweder aus ihm ausgetrieben oder in ihm zurückgehalten wird. Im Laufe der Zeit muss diese letztere Menge natürlich, wenn dann die Temperatur constant gehalten wird,

1) R. Bunsen, Gasometrische Methoden. p. 299.

2) N. Zuntz, Beiträge zur Physiologie des Blutes, Inauguraldissertation. Bonn 1868. p. 33 . 
gleich Null werden. Demnach soll man die Thiere dem Versuche erst unterwerfen, wenn sie sich annähernd in diesem Beharrungszustande befinden und den Versuch möglichst lange dauern lassen, wenn eine Temperaturveränderung des Froschkörpers stattgefunden hat.

Das ist nun freilich Alles sehr einfach und selbstverständlich. Wer meine Erörterung desshalb für überflüssig hält, dem gebe ich doch zu bedenken, dass Moleschott diese Verhältnisse nicht berücksichtigt hat, und dass alle Physiologen sich auf Moleschott's Versuche als Stütze für die Ansicht berufen, dass die Kohlensäurebildung der Kaltblüter mit der Temperatur wächst und dass diese Stütze eben wegen meiner Erörterung keine ist.

Den Sauerstoff hat Moleschott ebenso wenig als Marchand berücksichtigt.

Wenn ich also von älteren Beobachtungen absehe wie z. B. von Delaroche ${ }^{1}$ ), ebenso von einer höchst mangelhaften Arbeit von Treviranus ${ }^{2}$ ), so bleibt von guten messenden Versuchen nur übrig, was Regnault und Reis et ${ }^{3}$ ) auf Grund von nur 3 Analysen über die Respiration von Eidechsen bei verschiedenen Temperaturen melden - und diess ist durch den Winterschlaf complicirt :

Eidechsen absorbiren in Lethargie proKilo u. Stde. 0,0246 Gr. Sauerst. bei $7.3^{\circ} \mathrm{C}$.

"unvollkommen erwacht $\diamond " 0,0646 \gg, \$ 14.8^{\circ} \mathrm{C}$.

Dieser Versuch wird auch oft als Stütze für die Ansicht aufgeführt, dass die Oxydationsprocesse der Kaltblüter mit der Temperatur wachsen.

Leider ist dieser Versuch Regnault's getrübt durch peine enorme Stickstoffexhalation “, die wohl nur auf einem Beobachtungsfehler beruht, ferner dadurch dass die 3 Versuche in Intervallen von Monaten angestellt sind und keine Angabe vorliegt, ob die Thiere

1) Delaroche, Journal de physique LXXVII. 5. Das Journal de physique ist weder auf der Bonner $\mathrm{kgl}$. Bibliothek, noch überhaupt in Bonn oder in der Welt aufzutreiben, weshalb ich die Arbeit nicht kenne.

2) G. R. Treviranus, Versuche über das Athmen der niederen Thiere in Zeitschx. für Physiol. von Tiedemann, G. R. Treviranus und L. C. Treviranus. - Bd. IV. p. 1. 1831.

3) Regnault und Reiset, Recherches chimiques sur la Respiration des animaux des diverses Classes; - Annal. de Chimie et de Physique (3) XXVI. p. 483. 
etwa in der letzten Zeit viel gefressen hatten, als sie bei höherer Temperatur so hohe Respirationswerthe ergaben. Sicher waren sie lange nüchtern gewesen, als ihre Respiration in der Lethargie untersucht wurde.

Somit ruht unser Glaube, dass die Oxydationsprocesse der Kaltblïter mit der Temperatur zunehmen, nur auf einigen allgemeinen, allerdings - ich bekenne es - sehr werthvollen Notionen; darauf nämlich, dass die Kaltblüter im Winter beim Hungern wenig an Gewicht verlieren, dass Frösche bei niederer Temperatur immer unter Wasser bleiben können, nicht Luft zu schöpfen brauchen, ohne zu ersticken u.s.w. u.s.w. - Die interessanteste dieser Notionen ist, dass, wie von Spallanzani und Anderen gefunden worden ist, eine untere Temperaturgrenze existirt, welche die Thiere nicht tödtet, aber das Leben aufhebt, so dass Sauerstoffverbrauch und Kohlensäurebildung $=0$ für viele Monate. Herzschlag, Circulation, Athembewegung, Ernährung, Wachsthum haben aufgehört. Das ist die Grenze meiner Dissociationstemperatur '). Sobald diese Grenze nach aufwärts überschritten wird, beginnt das Leben wieder. Diese Grenze der Dissociationstemperatur scheint für verschiedene Geschöpfe nicht ganz gleichen Werth zu haben. Sie liegt nach Spallanzani ${ }^{2}$ ) für Schnecken bei $-1^{0} \mathrm{C}$ : Beim frischgelegten Vogelei, dessen Zustand einem tiefen Winterschlaf entspricht, scheint sie über $0^{0} \mathrm{zu}$ liegen. Eine der fundamentalsten Aufgaben ist es, diese Temperatur zu bestimmen!

1) Ich habe hier unter „Dissociationstemperatur « die Temperatur verstanden, bei der die Dissoeiation stattfindet. Oft wird aber darunter die Temperatur verstanden, unter welcher die Dissociation aufhört, und über welcher sie anfängt.

2) L. Spallanzani, Mémoires sur la respiration traduits par Senebier. 1803 . 